\title{
Estilos de liderazgo y su relación con la motivación en equipos de trabajo de bachillerato
}

\section{Leadership styles and their relationship with motivation in high school teams}

\author{
Ivette Alejandrina Quintal Marín \\ Ana Laura Contreras Abad \\ Marisa Hernández Tirado \\ Alejandro Nolasco Mena \\ Scarlett del Rosario Canto García \\ Rodrigo Enrique Cauich Sánchez \\ Universidad Autónoma de Yucatán
}

\section{Resumen}

Esta investigación tuvo como objetivo analizar la percepción de liderazgo de estudiantes de bachillerato acerca del líder seleccionado por ellos mismos en modalidad de equipo y su relación con la motivación que aquel les brinda como integrantes. Se analiza el trabajo en equipo en un contexto educativo porque es una de las herramientas más utilizadas en este ambiente. Se trató de una investigación empírica, cuantitativa, transversal y de campo, en un contexto educativo con estudiantes de dos preparatorias afiliadas a la Universidad Autónoma de Yucatán (UADY), con un total de 69 participantes. Los instrumentos fueron el test de Liderazgo de Kurt Lewin, que identifica tres estilos de liderazgo: autoritario, democrático y laissez-faire; y la Escala de Motivaciones (EM1), basada en el Modelo de McClelland, donde se utilizaron los tres tipos de motivación: poder, logro y filiación. Los resultados evidencian que se percibió con mayor frecuencia un estilo democrático, por lo tanto, se mostró una correlación positiva con la motivación de poder; dicho estilo de liderazgo hace que los integrantes se sientan parte importante del equipo, y fomenta su participación para lograr el objetivo esperado. De esta manera, la relación entre el liderazgo democrático y la motivación de poder resulta congruente con la teoría, ya que el estímulo principal para las personas con esta motivación, es su significación dentro del equipo. Se espera que el trabajo proporcione información para futuras investigaciones en el ámbito académico, con el fin de crear estrategias para incrementar la motivación en los estudiantes y su capacidad para trabajar en equipo.

Palabras clave: liderazgo, motivación, trabajo en equipo, bachillerato.

Nota del autor

Ivette Alejandrina Quintal Marín, Facultad de Psicología, Universidad Autónoma de Yucatán (UADY); Ana Laura Contreras Abad, Facultad de Psicología, UADY; Marisa Hernández Tirado, Facultad de Psicología, UADY; Alejandro Nolasco Mena, Facultad de Psicología, UADY; Scarlett del Rosario Canto García, Facultad de Psicología, UADY; Rodrigo Enrique Cauich Sánchez, Facultad de Psicología, UADY.

La correspondencia en relación con este artículo debe dirigirse a Ivette Alejandrina Quintal Marín, Facultad de Psicología, UADY, calle 60, número 491-A, por 57, colonia Centro, C. P. 97000, Mérida, Yucatán, México.

Dirección electrónica: ivetteqm94@gmail.com 


\begin{abstract}
This research had the target to analyze students leadership perception toward their own leader team, who also was selected by them, and their relation in high school students from two UADY affiliated schools. It is considered analyze teamwork in an educational context because it is one of the most used tools in this environment. It was an empirical, quantitative, transversal and field research, made in an educational context with 69 participants. The two instruments used were: Kurt Lewin leadership test, which identifies three kind of leadership: authoritarian, democratic and laissez-faire; and the motivations scale EM1, based on the McClelland model by Álvarez, which identifies three motivational types: power, achievement and affiliation. The results evidence show that the most frequently perception was toward the leader with democratic style, with a positive correlation of power motivation. This leadership style makes feel the members fundamental encouraging their participation to achieve their goal, therefore this relationship results congruent with theory, because the principal stimulation to people with this motivation is that they are important to the team. It is expected that the work contributes providing information for future research on the academic area to create strategies for increasing student motivation.
\end{abstract}

Keywords: leadership, motivation, teamwork, high school.

Actualmente el trabajo en equipo es una herramienta relevante para lograr la integración necesaria en los estudiantes la obtención de resultados, también nos permite desarrollar habilidades como la tolerancia, el respeto y la comunicación efectiva entre diferentes personas y sus contextos. Muchas veces el trabajo en equipo resulta una carga para el estudiante, ya que no se trabaja de manera colaborativa, cada integrante realiza su parte y no se involucra con las de los demás, limitándose entonces a un trabajo en grupo. Su objetivo principal, es, por consiguiente, obtener una calificación numérica alta y culminar con la tarea o proyecto, perdiendo de vista la calidad y el compromiso que debe estar inmerso en un trabajo en equipo (Ángeles, 2009).

Según Katzenbach y Smith (2011), un equipo es un grupo pequeño de personas que tiene un objetivo en común y metas de desempeño, que mediante habilidades complementarias se comprometen a lograr dicho objetivo en conjunto. Las personas que forman un equipo interaccionan de forma verdadera, fortaleciéndose los unos a los otros, es entonces que mediante una interacción eficaz de todos los individuos se crea y aplica un valor añadido que mejora la actuación del equipo (Martínez \& Salvador, 2005). Al interactuar los miembros de un equipo, podemos observar que las conductas individuales influyen entre sí para desarrollar una nueva conducta que aparece como el comportamiento del conjunto.

El Modelo Educativo para la Formación Integral (MEFI) de la Universidad Autónoma de Yucatán (UADY) menciona con gran énfasis que, el alumno egresado de bachillerato deberá contar con las habilidades necesarias 
para trabajar correctamente en equipo. Así, el MEFI enfatiza la importancia de desarrollar en los egresados del nivel medio superior las capacidades que les permitan responder a los diversos contextos sociales, tanto locales como nacionales e internacionales. El trabajo en equipo no es sólo una herramienta académica, ya que posteriormente las capacidades desarrolladas con esta estrategia pueden ser utilizadas en asuntos profesionales, es importante ponerla en práctica desde bachillerato y así fomentar las habilidades necesarias (UADY, 2012).

Para Ángeles (2009), un equipo efectivo es aquel que soluciona problemas de manera integrada, corresponde a un cuerpo de personas donde se establecen los objetivos a lograr de manera efectiva, cada uno de sus integrantes muestra el compromiso y la responsabilidad inherente a su rol dentro del equipo, colaboran para lograr vínculos armoniosos con una sana convivencia fuera y dentro de un salón de clases y superar las debilidades para integrarse en una función colaborativa, en ella se escuchan opiniones diferentes y se integran, o bien, es seleccionada la que mejor fundamente la parte lógica y racional del proyecto.

Lussier y Achua (2005) mencionan que una de las características más importantes del trabajo en equipo es el liderazgo. Robbins y Judge (2013) lo definen de la siguiente manera: "la habilidad de influenciar a un grupo de personas hacia un conjunto de metas u objetivos" (p. 368).

Un equipo necesita de un líder efectivo, y que este mismo maneje su autoridad de una manera democrática. Asimismo, no se puede negar el uso del poder como influyente en las ideas y gestiones de los integrantes (Ramos, 2005). Es de vital importancia que el equipo participe en la definición de los objetivos, para ello es necesario que el líder genere responsabilidad y así cada uno aporte lo necesario. Debe, además, dirigir la comunicación para que todos sean partícipes de los resultados finales; manejar los conflictos que puedan surgir; y ostentar la representación al defender al equipo contra cualquier situación (Acosta, 2011).

A partir de un experimento llevado a cabo por Lewin (como se cita en Torrecilla, 2006), se explica cómo es condicionado el comportamiento de los individuos según diversos tipos de liderazgo. Se definieron tres estilos principales de liderazgo: liderazgo autoritario, liderazgo democrático, liderazgo laissez-faire. (Torrecilla, 2006).

Otro factor importante, determinante para el trabajo en equipo, es la motivación. Robbins y Judge (2013) la describen como "los procesos que inciden en la intensidad, dirección y persistencia del esfuerzo que realiza una persona para alcanzar un objetivo" (pp. 202). McClelland (como se citó en Barreiro \& Carreño, 2011), distingue tres tipos de motivación: motivación de logro, motivación de poder y motivación de filiación.

Lussier y Achua (2005) mencionan que una relación existente entre el liderazgo y la motivación sugiere que la capacidad para motivar a otros, o poseer habilidades para motivar, es 
fundamental para el éxito del liderazgo. Por lo cual se puede concluir que el líder cumple una función fundamental para la motivación en la realización de las respectivas tareas, y el mantenimiento del interés de los miembros del equipo para llegar a las mismas. De acuerdo a Acosta (2011), el liderazgo y la motivación deben ser analizados como conductas, ya que se trata del comportamiento de un individuo en un equipo y situación específica, cuya consecuencia es la influencia positiva o negativa sobre el equipo.

En la presente investigación se analiza la relación entre los diversos estilos de liderazgo planteados por Kurt Lewin (como se cita en Torrecilla, 2006) y los tipos de motivación de acuerdo con el modelo Motivacional de McClelland (1961). Es importante mencionar que existen investigaciones en las cuales el trabajo en equipo y sus variables son objeto de estudio, más en su mayoría fueron realizadas en un contexto laboral, y no en uno educativo, como es el caso del presente estudio.

La investigación se enfoca en la motivación de los miembros de un equipo en relación con la percepción que ellos mismos tienen acerca de su líder en cuanto a su estilo de liderazgo. El equipo es considerado como el contexto donde ocurre dicha relación. Debido a que en áreas de la educación, organizacional o laboral se utiliza el trabajo en equipo para la realización de tareas y proyectos, se puede explicar por qué la motivación tiene una gran influencia en el desarrollo del mismo. De acuerdo a Chang (2010), conocer la motivación para el trabajo es sumamente importante y resulta útil para mejorar el rendimiento y lograr las metas planeadas.

Este tema es de vital importancia, debido a que el trabajo en equipo es una de las estrategias más utilizadas por los profesores dentro de las preparatorias afiliadas a la UADY, y, en general, resulta ser una herramienta que se utiliza con frecuencia para la realización de tareas (UADY, 2012).

\section{Método}

La investigación parte de la siguiente pregunta: ¿Cómo se relaciona la percepción que tienen los integrantes de un equipo acerca del estilo de liderazgo de su líder con la motivación que dichos miembros del equipo poseen?

\section{Objetivo general}

Analizar la relación entre los estilos de liderazgo percibidos y los diversos tipos de motivación que mantiene cada miembro en equipos de estudiantes de preparatorias afiliadas a la UADY.

\section{Objetivos específicos}

- Identificar los diversos estilos de liderazgo percibidos por los miembros del equipo.

- Identificar los diversos tipos de motivación presentes en cada integrante de los equipos.

- Encontrar la relación entre los etilos de liderazgo percibidos y los tipos de moti- 
vación encontrados en miembros de los equipos.

Para responder a dicha pregunta de investigación y cumplir con el objetivo general se plantearon las siguientes hipótesis:

H1 (1): El liderazgo democrático se relaciona con la motivación de filiación en los miembros del equipo.

H1 (2): El liderazgo autoritario se relaciona con la motivación-logro en los miembros del equipo.

H1 (3): El Liderazgo laissez-faire se relaciona con la motivación de poder en los miembros del equipo.

\section{Variables}

- Estilo de liderazgo: Abarca desde cómo se relacionan los líderes con otros dentro y fuera de la organización, hasta como se ven a sí mismos y su posición, si son o no exitosos como líderes (Gardner, 1991).

- El liderazgo autoritario: El líder concentra todo el poder y la toma de decisiones. Es un ejercicio del liderazgo unidireccional, donde los seguidores obedecen las directrices que marca el líder.

- El liderazgo democrático: Se basa en la colaboración y participación de todos los miembros del equipo.

- El liderazgo laissez-faire: El líder no ejerce su función, no se responsabiliza del equipo y deja a éste a su propia iniciativa.

- Motivación: "los procesos responsables del deseo de un individuo de realizar un gran es- fuerzo para lograr los objetivos organizacionales, condicionado por la capacidad del esfuerzo de satisfacer alguna necesidad individual" (Robbins \& Judge, 2013, p. 392).

- Motivación de logro: el impulso de tener éxito. Hace que los mismos individuos se pongan metas y objetivos. Este tipo de personas tienen una gran necesidad de desarrollar varias actividades. Las personas dirigidas por este motivo quieren la excelencia, hacen un trabajo bien realizado, tienen y adquieren sus responsabilidades y necesitan una retroalimentación persistente sobre sus acciones.

- Motivación de poder: la necesidad de ser influyente y controlador sobre otras personas o grupos, además de tener reconocimiento por parte de ellas. A las personas motivadas por esto les agrada que se les tenga en cuenta y sean importantes, desean tener progresivamente prestigio y posición social.

- Motivación de filiación: el deseo de tener relaciones interpersonales amistosas con los demás y cercanas, además de formar parte de un equipo. A este tipo de individuos les agrada normalmente ser populares, tratar con los demás, ya que no les gusta el trabajo individual y satisface trabajar en equipo y ayudar a los demás (Barreiro \& Carreño, 2011).

\section{Participantes}

La investigación se realizó en un contexto escolar en la Ciudad de Mérida, Yucatán. El tipo 
de muestra utilizado fue no probabilístico intencional, ya que se establecieron parámetros tales como que los participantes pertenezcan a instituciones afiliadas a la UADY, y, principalmente, que utilicen proyectos o tareas a largo plazo en equipos de trabajo.

La población total se determinó a partir de dos preparatorias privadas; estuvo conformada por un grupo por cada grado escolar, con un total de seis grupos: dos de primer grado, dos de segundo grado y dos de tercer grado con un contenido variado de estudiantes en cada grupo. Los participantes fueron jóvenes estudiantes que están cursando dicho nivel educativo. La muestra estuvo conformada por $50.72 \%$ de los participantes de sexo femenino y $49.27 \%$ de sexo masculino con edades entre 15 y 21 años, en su mayoría católicos, todos solteros. Sumaron un total de 69 participantes.

\section{Instrumentos}

Los instrumentos utilizados fueron dos: el Test de Liderazgo de Kurt Lewin y la Escala de Motivaciones (EM1), basada en el Modelo de McClelland desarrollado por Álvarez (2012). En la primera prueba se presentan 33 enunciados en los cuales se tiene que indicar si se está de acuerdo o en desacuerdo. Este test busca identificar tres estilos de liderazgo: estilo autoritario, estilo democrático, estilo laissez-faire. A este instrumento se le hicieron cambios en algunas palabras con fines de contextualización "escuela" en vez de "trabajo".
El estudio realizado por Al-Khasawneh y Moh'd (2012) evidencia el coeficiente de confiabilidad Alfa de Cronbach de la escala de 0.8 y menciona que los investigadores verificaron la validez del instrumento, al presentarlo ante un número de personal académico en universidades Jordanias (Al-Khasawneh \& Moh'd, 2012).

Por su parte, el segundo instrumento consiste en una escala tipo Likert, dividida en tres subescalas: poder, logro y filiación. La subescala de logro, cuenta con 28 ítems; la subescala de poder con cuatro ítems; y la subescala de filiación con trece ítems. Cada uno de ellos debe contestarse en un formato con cinco opciones de respuesta: en Desacuerdo totalmente (1), Casi totalmente en desacuerdo (2), En ocasiones de acuerdo (3), Casi totalmente de acuerdo (4), De acuerdo totalmente (5). El instrumento consta de un total de 44 ítems. El instrumento original constaba de cuatro tipos de motivaciones; además de las tres básicas de McClelland, incluía una cuarta llamada: reconocimiento, sin embargo, al momento de extraer los resultados no se tomó en cuenta este tipo de motivación, ya que la investigación está basada en la Teoría de las necesidades de McClelland (1961), quién solo menciona tres tipos de motivación. Los resultados arrojados por el estudio de Álvarez (2012) evidencian razones de confiabilidad de tipo estadístico con un coeficiente de Alfa de Cronbach de la escala de 0.87 y una validez de constructo (Álvarez, 2012). 


\section{Procedimiento}

La aplicación de los instrumentos fue de acuerdo al número total de alumnos en cada grupo. Estos instrumentos fueron aplicados a los estudiantes entre los meses de noviembre y diciembre del 2014 con una duración aproximada de 20 minutos por salón. La aplicación se realizó en horario de clase y en presencia del maestro de la respectiva clase.

Momentos antes de la aplicación de los instrumentos, se otorgaron las instrucciones a los estudiantes para la resolución del primer instrumento, el Test Liderazgo de Kurt Lewin, con el objetivo de que los estudiantes tuviesen claro el contexto que calificarían, es decir, un trabajo en equipo. La principal instrucción hacia los equipos fue mencionar al líder en curso o determinar uno, en caso de que aún no se hubiese establecido uno. Para ayudar a los participantes a saber qué evaluar, se les preguntó qué integrante ha sido quien se ha encargado de reunir a los integrantes del equipo y tomar ciertas decisiones importantes para el cumplimiento de las tareas, incluidas dentro del proyecto. La persona elegida o autodenominada líder pasó al frente como representante del equipo, más tarde, fue aplicado el instrumento de liderazgo al resto de los integrantes. Posteriormente, se administró a los alumnos la Escala de Motivación de McClelland (1961).
Finalizada la aplicación de ambas pruebas, se agradeció a los participantes por su colaboración, seguidamente a los profesores presentes en las aulas de clases $\mathrm{y}$, por último, a las autoridades responsables de las preparatorias. Después de la aplicación de los instrumentos se procedió al análisis de datos. Las respuestas se integraron a los instrumentos en el software Excel, y los datos de los instrumentos fueron trasladados al Programa Estadístico para Ciencias Sociales (SPSS, por sus siglas en inglés, versión 21.0), donde se utilizó una correlación de Pearson de las variables: estilos de liderazgo y tipo de motivación; adicionalmente fueron calculadas estadísticas descriptivas, medias, modas, desviaciones estándar para cada variable y frecuencias de datos sociodemográficos.

\section{Resultados}

En las siguientes tablas se muestran los datos obtenidos. En la tabla 1 se encuentran las frecuencias de las percepciones de liderazgo. Se observa que la mayoría de los sujetos percibe a su líder con un estilo de liderazgo democrático con una frecuencia de 57. Igualmente, se puede observar que algunos estilos tuvieron igualdad en puntuación. 
Tabla 1

Frecuencias de las percepciones de los estudiantes sobre liderazgo

\begin{tabular}{ll}
\hline \multicolumn{1}{c}{ Sujetos } & Frecuencia \\
\hline Sujetos que perciben un líder predominantemente autoritario & 3 \\
Sujetos que perciben un líder predominantemente democrático & 57 \\
Sujetos que perciben un líder predominantemente laissez-faire & 3 \\
Sujetos que perciben un líder predominantemente autoritario-democrático & 3 \\
Sujetos que perciben un líder predominantemente democrático/laissez-faire & 3 \\
\hline
\end{tabular}

Por otro lado, en la tabla 2, se encuentran los tipos de motivación identificados en los sujetos. Se observa que la mayoría de los participantes se identificó con una motivación filiativa con una frecuencia de 33. Se logra observar, asimismo, que un participante se identificó con más de una motivación.

La evidencia en la tabla 3 muestra que en el estilo de liderazgo democrático se encontró una correlación de Pearson positiva-significativa con motivación de poder $r=0.024$. Por su parte, el estilo de liderazgo autoritario se encontró una correlación negativa-significativa con la motivación al logro $r=0.024$ y la motivación filiativa $r=0.007$; finalmente, el estilo de liderazgo laissez-faire mostró una correlación significativa con el tipo de motivación filiativa $r=0.024$.

Tabla 2

Frecuencias de las percepciones de los estudiantes sobre la motivación

\begin{tabular}{ll}
\hline \multicolumn{1}{c}{ Sujetos } & Frecuencia \\
\hline Sujetos con mayor motivación al logro & 18 \\
Sujetos con mayor motivación al poder & 17 \\
Sujetos con mayor motivación filiativa & 33 \\
Sujetos con mayor motivación poder-filiativa & 1 \\
\hline
\end{tabular}

Tabla 3

Correlaciones de las variables liderazgo-motivación

\begin{tabular}{lllll}
\hline Variable & & Logro & Poder & Filiativa \\
\hline \multirow{2}{*}{ Autoritario } & Correlación de Pearson & -0.271 & -0.162 & -0.324 \\
& Sig. (Bilateral) & $\mathbf{0 . 0 2 4}$ & 0.183 & $\mathbf{0 . 0 0 7}$ \\
Democrático & Correlación de Pearson & 0.081 & 0.272 & 0.163 \\
& Sig. (Bilateral) & 0.509 & $\mathbf{0 . 0 2 4}$ & 0.18 \\
\multirow{2}{*}{ Laissez-faire } & Correlación de Pearson & 0.1 & -0.136 & 0.271 \\
& Sig. (Bilateral) & 0.416 & 0.265 & $\mathbf{0 . 0 2 4}$ \\
\hline
\end{tabular}


Se rechaza la hipótesis H1 (1), "el liderazgo democrático se relaciona con la motivación de filiación en los miembros del equipo", dado que no se encontró correlación significativa; $\sin$ embargo, de acuerdo con los datos obtenidos se identificó una correlación entre este estilo de liderazgo y la motivación de poder. En cuanto a la hipótesis H1 (2), "el liderazgo autoritario se relaciona con la motivación logro en los miembros del equipo", no fue rechazada, ya que se obtuvo una correlación inversamente proporcional, es decir, a mayor liderazgo autoritario menor motivación de logro. De igual forma se determinó que dicho estilo de liderazgo tiene una correlación con la motivación filiativa inversamente proporcional, o bien, a mayor liderazgo autoritario menor motivación filiativa. Para concluir, la hipótesis H1 (3), "el liderazgo laissez-faire se relaciona con la motivación de poder en los miembros del equipo" se rechaza, pues se correlaciona con la motivación filiativa y no con la motivación de poder.

\section{Discusión}

De acuerdo con los datos obtenidos en esta investigación fueron analizados los resultados. Primeramente, es importante mencionar que se ratificó lo citado en el marco teórico, donde Lussier y Achua (2005) sugieren una relación existente entre el liderazgo y la motivación, ya que en los resultados se muestra que diversos estilos de liderazgo se correlacionan con algún tipo de motivación.
En cuanto a los resultados generales, es evidente que la mayoría de los participantes percibió a su líder con un estilo de liderazgo democrático. Esto es favorable para los estudiantes, dado que la teoría de Kurt Lewin (como se cita en Torrecilla, 2006) sostiene que así se genera un mejor funcionamiento dentro del equipo, de acuerdo con sus características (Torrecilla, 2006).

En el estilo de liderazgo democrático los integrantes son tomados en cuenta como parte fundamental del equipo; además de fomentar en ellos la participación en busca del mismo objetivo (Torrecilla, 2006), resulta congruente que exista una relación con la motivación de poder, ya que según Barreiro y Carreño (2011), lo que estimula a las personas con este tipo de motivación es que las tomen en cuenta y sean importantes dentro de un grupo social, lo cual sucede dentro de dichos equipos.

Por otra parte, de acuerdo a Torrecilla (2006), un líder con características de estilo autoritario se enfoca hacia resultados favorables, marcando disciplina y manteniendo distancia hacia el resto de los integrantes; evita, además, involucrarse emocionalmente con ellos, por lo cual resulta congruente con los resultados que este tipo de líder no fomente una motivación filiativa. De igual manera, se obtuvo que la variable percepción de estilo de este liderazgo tiene una correlación inversamente proporcional con la motivación para el logro, es decir, cuando los estudiantes perciben a su líder con un estilo autoritario hay una relación menor de motivación 
a logro en ellos, esto puede deberse a que este estilo de liderazgo suele dar órdenes y liderar de manera unidireccional, en cuanto a poder y toma de decisiones, disminuyendo la probabilidad de que los integrantes tengan una orientación hacia el éxito, tracen sus propias metas y seguirlas.

La variable percepción de estilo de liderazgo laissez-faire tuvo una correlación con la motivación filiativa. Esta correlación concuerda con la teoría, pues el estilo de liderazgo anteriormente mencionado se refiere a un líder que mantiene una relación cercana entre los integrantes del equipo, sin tener que fomentar la autoridad y la disciplina; es un líder despreocupado por la distribución del trabajo e indiferente ante los resultados del mismo. De acuerdo con la Teoría de las necesidades de la motivación, las características de la motivación filiativa son las interacciones con los demás integrantes y los vínculos que se pueden llegar a tener con éstos (Barreiro \& Carreño, 2011).

En conclusión, se puede observar la importancia que tiene el estilo de liderazgo que ejerce el líder en el equipo en cuanto a la motivación de los integrantes. Y aun cuando la mayoría de los participantes ha percibido a su líder con estilo de liderazgo democrático, el funcionamiento de los equipos de bachillerato no siempre resulta ser el mejor. Por esto se considera importante que las instituciones incorporen un modelo o plan de estudios que fomente el trabajo en equipo con énfasis en las habilidades de liderazgo. Dado esto, se recomiendan futuras investigaciones sobre el tema, con referencia el presente trabajo.
Igualmente, con los resultados obtenidos se podría facilitar la aplicación de estos instrumentos con miras al estudio de la relación liderazgo-motivación en ambientes escolares y así profundizar más en ello. Actualmente, no hay muchos estudios sobre este tema $y$, en específico, de ahí que el estudio de tal relación abarca su aplicación en ambientes organizacionales para ampliar teorías o conocer con profundidad este fenómeno. Este trabajo contribuirá al proporcionar información para futuras investigaciones en el ámbito académico con el fin de crear estrategias para incrementar la motivación en los estudiantes, así como poder hacer investigaciones en los diferentes niveles académicos, desde el nivel básico primaria hasta un nivel superior.

El presente trabajo fue de carácter exploratorio, se consideraron teorías clásicas y se corroboró que estas teorías son funcionales en la actualidad, aunque igualmente se observó que pudieran tener más clasificaciones de las que las teorías abordan, por lo tanto, se propone ahondar en este tema con teorías recientes y con el objetivo de ampliarlas. Del mismo modo, debe mencionarse que esta investigación es un tema relevante e importante en el que hay que profundizar, por ello se sugiere extender el número de participantes a una población mayor, abarcar otras zonas geográficas e incluir varias escuelas que no estén afiliadas a la UADY, para realizar así correlaciones y comparaciones.

Durante la elaboración de la presente investigación se presentaron diversas 
limitaciones, como el número de participantes que se tomó para su realización. Aunque fueron tomadas las poblaciones totales de dos escuelas, en ellas había pocos estudiantes, aun cuando se constituyó una muestra que resultó adecuada y con resultados significativos. Otra traba principal en la elaboración se debió a la falta de literatura en el área educativa, dado que investigaciones encontradas para la elaboración del marco teórico eran referentes al área organizacional. Cuando se habla de liderazgo no siempre se piensa en escuelas, generalmente, la mayoría de las investigaciones se centra en el anterior ámbito.

Otra de las restricciones encontradas en la realización de la presente investigación la conformó la idea de tomar como población a las preparatorias de la UADY, principalmente, la Preparatoria N. 1 y Preparatoria N. 2, por ser estas las más reconocidas en Mérida, gracias a su apego al Modelo MEFI (2010). Debido a cuestiones administrativas en dichas escuelas estas no pudieron formar parte de la población estudiada y se eligieron escuelas que estuvieran incorporadas a la UADY. Otro de los inconvenientes encontrado, durante la aplicación de instrumentos, fue que algunos participantes se mostraron desinteresados al contestarlos, y no les daban importancia.

\section{Referencias}

Acosta, J. (2011). Trabajo en equipo. Madrid, España: ESIC.

Al-Khasawneh A., \& Moh'd S. (2012). The impact of leadership styles used by the academic staff in the jordanian public universities on modifying students' behavior: A field study in the northern region of Jordan. International Journal of Business and Management, 8(1). Recuperado de http:// dx.doi.org/10.5539/ijbm.v8n1p1

Álvarez, R. (2012) Escala de motivación adolescente (EM1) basada en el Modelo Motivacional de McClelland. Red de Revistas Cientificas de América Latina, el Caribe, España y Portugal, 7, 128-143. Recuperado de http://www.redalyc.org/ articulo.oa? id=139025258006

Ángeles, S. T. (2009). Perfil del estrés académico en alumnos de licenciatura en psicología de la Universidad Autónoma de Hidalgo en la escuela superior de Actopan. (Tesis inédita de licenciatura). Universidad Autónoma del Estado de Hidalgo, México.

Barreiro, C., \& Carreño, O. (2011). El trabajo en equipo. AMF Revista en Medicina de Familia, 7(2).

Chang, A. L. (2010). Estudio de la motivación laboral y el conocimiento de la necesidad predominante según la teoría de las necesidades de McClelland, en los médicos del hospital nacional Arzobispo Loayza (Tesis de maestría publicada). Universidad Nacional Mayor de San Marcos, Lima, Perú. 
Gardner, J. (1991). El liderazgo. Buenos Aires, Argentina: Grupo Ed. Latinoamericano.

Katzenbach, J., \& Smith, D. (2011). The discipline of teams. Harvard Business, 71(2), 111-120.

Lussier, R., \& Achua, C. (2005). Liderazgo, teoría, aplicación y desarrollo de habilidades. México: Thomson.

McClelland, D. C. (1961). The achieving society. Princeton, NJ: Van Nostrand Co.

Martínez, M., \& Salvador M. (2005). Aprender a trabajar en equipo. Madrid, España: Paidós.

Ramos, A. (2005). Mujeres y liderazgo: Una nueva forma de dirigir. Valencia, España: Universidad de Valencia.

Robbins, S., \& Judge T. (2013). Comportamiento Organizacional. México: Pearson.

Torrecilla, M. F. J. (2006). Una dirección escolar para el cambio: Del liderazgo transformacional al liderazgo. Revista Iberoamericana sobre Cambio y Eficacia Escolar, 4, 11-24.

Universidad Autónoma de Yucatán (UADY). (2012). Modelo educativo para la formación integral. Recuperado de http://www.dgda. uady.mx/media/docs/mefi_dgda.pdf

Recibido el 4 de junio de 2015

Revisado el 25 de julio de 2015 Aceptado el 4 de septiembre de 2015 